NASA Technical Memorandum 107604

\title{
SYSTEM IDENTIFICATION FROM CLOSED- LOOP DATA WITH KNOWN OUTPUT FEEDBACK DYNAMICS
}

Minh Phan, Jer-Nan Juang, Lucas G. Ilorta, and Richard W. Longman

April 1992

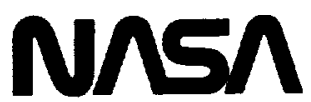

National Aeronautics and

Space Administration

Langley Research Center

Hampton, Virginia 23665

$$
\begin{aligned}
& \text { (NASA-TM-107004) SYSTEM IDFNTIFICATION FROM } \\
& \text { CL JSF -LORP SATA WITH KNUWN OUTPUT FEEDBACK } \\
& \text { CYNAMILS (NASA) } 340 \text { CSCL } 20 K
\end{aligned}
$$





\title{
System Identification from Closed-Loop Data with Known Output Feedback Dynamics
}

\author{
Minh Phan," Jer-Nan Juang, ${ }^{* *}$ Lucas G. Horta, ${ }^{+}$and Richard W. Longman ${ }^{++}$ \\ NASA Langley Research Center, Hampton, VA 23665
}

\begin{abstract}
This paper presents a procedure to identify the open-loop system when it is operating under closedloop conditions. First, closed-loop excitation data are used to compute the system open-loop and closed-loop Markov parameters. The Markov parameters, which are the pulse response samples, are then used to compute a state space representation of the open-loop system. Two closed-loop configurations are considered in this paper: The closed-loop system can either have a linear output feedback controller or a dynamic output feedback controller. Numerical examples are provided to illustrate the proposed closed-loop identification method.
\end{abstract}

\section{Introduction}

The problem of identification of the open-loop system when it is operating under closed-loop conditions is considered. This is a problem of considerable practical importance. There are several instances when this is needed. For example, consider the case when an open-loop model of the system is required, but the system is operating in closed-loop, and it is not possible to remove the existing controller for open-loop identification. Even when it is possible to remove the existing controller for open-loop identification, it may not be desirable to do so. This is the case when the open-loop system is only marginally stable or unstable, and the existing controller is needed to ensure overall system stability. An open-loop model of the system may be required for purpose of analysis or controller re-design. It is generally not possible to identify the open-loop system simply by measuring the system output and the actual input during closed-loop operation because

\footnotetext{
*Engineer, Lockheed Enginecring \& Sciences Company, Hampton, VA., Member AIAA.

** Principal Scientist, Spaccoraft Dynamics Branch, Fcllow AIAA.

+ Acrospace Engincer, Spacecraft Dynamics Branch, Mcmber AIAA.

++ Professor of Mechanical Enginecring, Columbia Universily, New York, NY., Fellow AIAA.
} 
such input is usually not rich enough for identification. Additive input excitation during closedloop operation is necessary to identify the open-loop system. However, the excitation must be added in such a way that it does not affect overall system stability that is provided by the existing controller.

The paper considers the following closed-loop identification problems: First, the identification of a system having an existing linear output feedback controller is formulated. This is a basic case, upon which several extensions can be made. Second, if the open-loop system has a direct transmission term, the mathematical problem becomes slightly more complicated, and this case is treated next. Third, a solution to the more general problem where closed-loop system has output feedback dynamics is presented. Fourth, since the open-loop system may include known input and/or output filters in addition to the plant, it is sometimes desirable to extract the plant dynamics alone. Mathematically, this is the case of recovering the dynamics of one system from the combined dynamics of two cascading linear systems when the other system is known. Finally, numerical examples are provided to illustrate each of the developed identification procedures.

The closed-loop identification method developed here is based on the concept of an observer. Of importance are certain basic algebraic relations between the Markov parameters of the observer, and those of the closed-loop and open-loop system. The use of Markov parameters in system identification is discussed in Ref. 1 . The observer concept has been used previously in developing several open-loop identification techniques. ${ }^{2-8}$ Even though the closed-loop system identification method developed in this paper is derived within a deterministic framework, the resultant identification equations have the same structure as those developed previously for observer/Kalman filter identification (OKID). The readers are referred to Ref. 4 and Ref. 7 for an investigation of the stochastic properties of identification equations possessing this structure in the presence of process and measurement noises. From the identified open-loop system Markov parameters, a state space realization of the system is obtained by the Eigensystem Realization Algorithm (ERA), Ref. 10. In the presence of noises, a variance based confidence criterion for ERA identified modal parameters is developed in Ref. 11.

In this paper, the output feedback dynamics is assumed known. This requirement makes the problem mathematically well-posed. If feedback dynamics is not known, then the feedback signal is required to be known, which is the case treated in Ref. 12 for a closed-loop system possessing a full state feedback structure. 


\section{Problem Statement}

Consider a linear multivariable system expressed in state space format as

$$
\begin{aligned}
x(i+1) & =A x(i)+B u(i) \\
y(i) & =C x(i)
\end{aligned}
$$

where $x(i) \in R^{n}, y(i) \in R^{q}, u(i) \in R^{m}$, i.e., $n$ denotes order of the system, $q$ is the number of outputs, and $m$ is the number of inputs. The system has an existing linear output feedback controller with a gain $F$. For purpose of identification, an additional excitation $v(i)$ is injected to the control input, and the total input to the closed-loop system becomes

$$
u(i)=F y(i)+G v(i)
$$

which yields the following system of the form

$$
\begin{aligned}
x(i+1) & =(A+B F C) x(i)+B G v(i) \\
y(i) & =C x(i)
\end{aligned}
$$

For simplicity, let $A_{c}$ denote the closed-loop system matrix,

$$
A_{c}=A+B F C
$$

The additive excitation input vector $G v(i)$ and the corresponding output of the closed-loop system $y(i)$ are known. The existing linear feedback gain $F$ is assumed to be known. See Fig. 1 for a schematic diagram of the closed-loop system. The objective of the problem is to obtain a state space model of the open-loop system, denoted by the set $(A, B, C)$, from input-output data of the closed-loop system.

Furthermore, this is to be accomplished by first obtaining the Markov parameters of the open-loop system, $Y(i)=C A^{i-1} B$. A state space model of the system can then be computed from these Markov parameters using a realization method, Ref. 10. Subsequent developments that treat several extensions of this basic problem will be considered. 


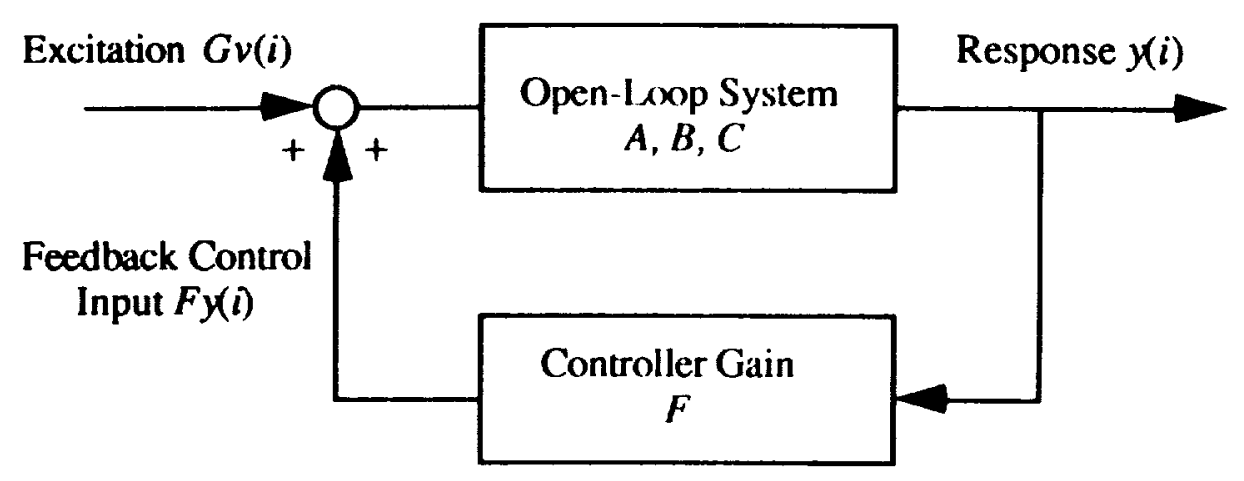

Fig. 1: Schematic diagram of the closed-loop system

\section{Mathematical Formulation}

The mathematical formulation consists of the following developments. The input-output description of the closed-loop system is described in terms of an observer whose Markov parameters can be computed from closed-loop data. Algebraic relations between the Markov parameters of the observer and those of the closed-loop and open-loop system are derived. From these relations, the open-loop system dynamics can be recovered from the computed observer Markov parameters and knowledge of the feedback gain. For clarity of presentation, the formulation is done first for the case without a direct transmission term, then extended to the case with a direct transmission term. This is because inclusion of the transmission term at the beginning would complicate the algebra without altering it fundamentally. Also, the case of the closed-loop system with a linear output feedback controller is treated first. The case with a dynamic controller then follows. Further extensions to the situation when the open-loop plant includes input or output filters are considered.

\section{The Closed-Loop System and Its Associated Observer}

This section introduces the concept of an observer as a intermediate step in solving for the Markov parameters of the open-loop system. First, note from Eq. (3) that the additive excitation signal $v(i)$ does not affect the overall closed-loop system stability that is being provided by the feedback controller since it does not alter the existing closed loop system matrix $A_{c}=A+B F C$. To solve for the Markov parameters of the closed-loop system in Eq. (3), an observer is introduced to this set of equations. This is accomplished by adding and subtracting the term $M y(i)$ to the right hand side of the state equation in Eq. (3), 


$$
\begin{aligned}
x(i+1) & =A_{c} x(i)+B G v(i)+M y(i)-M y(i) \\
& =\left(A_{c}+M C\right) x(i)+B G v(i)-M y(i)
\end{aligned}
$$

Define the following quantities

$$
\bar{A}_{c}=A_{c}+M C, \quad \bar{B}=[B,-M], \quad z(i)=\left[\begin{array}{c}
G v(i) \\
y(i)
\end{array}\right]
$$

Then the original closed-loop system can be expressed as

$$
\begin{aligned}
x(i+1) & =\bar{A}_{c} x(i)+\bar{B} z(i) \\
y(i) & =C x(i)
\end{aligned}
$$

It has been shown in Ref. 3 or 5 that the above operation is equivalent to introducing an observer to the system if the state $x(i)$ is considered as an observer state, and the matrix $M$ can be considered as an observer gain. In this particular case, it is an observer of the closed-loop system given by Eq. (3). It is important to note at this point that one does not have to know $M$ explicitly at the beginning, but rather it will be specified implicitly through the stability requirement for $\bar{A}_{c}=A_{c}+M C$. Assuming zero initial conditions for $x(i)$, the input-output description of the above system is

$$
y(i)=\sum_{\tau=0}^{i-1} C \bar{A}_{c}^{i-\tau-1} \bar{B}_{2}(\tau)
$$

If the system is made asymptotically stable by having a matrix $\bar{A}_{c}$ such that the Markov parameters $C \bar{A}_{c}^{p} \bar{B}, C \bar{A}_{c}^{p+1} \bar{B}, C \bar{A}_{c}^{p+2} \bar{B}, \ldots$ can be neglected for some $p$, then at time steps $i \geq p$, the inputoutput description can be approximately described by a reduced set of $p$ Markov parameters $C \bar{B}, C \overline{A_{c}} \bar{B}, \ldots, C \bar{A}_{c}^{p-1} \bar{B}$. The freedom in $M$ can be used to make the observer equation stable by placing its poles. Various cases where the prescribed poles may be real, complex, or deadbeat are studied in Ref. 5, and more extensively in Ref. 8 . In the case where $M$ is a deadbeat observer gain, the input-output relation of the above system can be expressed in terms of a finite set of observer Markov parameters as 


$$
y(i)=\sum_{\tau=1}^{p} \bar{Y}_{c}(\tau) z(i-\tau)
$$

where the observer Markov parameters $\bar{Y}_{c}(i), i=1,2, \ldots, p$, are defined as

$$
\bar{Y}_{c}(i)=C \bar{A}_{c}^{i-1} \bar{B}
$$

and $\bar{Y}_{c}(i)=0$ for $i=p+1, p+2, \ldots$ Suppose that a set of $N$ measurements of the closed-loop system $y(i)$ and $u(i), i=0,2, \ldots, N-1$, given in Eq. (3) is available. Assuming zero initial conditions for the moment, the input-output relation for the set of measurements can be written in matrix form as

$$
y=\bar{Y}_{c} V
$$

where

$$
\begin{aligned}
& y=\left[\begin{array}{lllllll}
y(1) & y(2) & \cdots & y(p) & y(p+1) & \cdots & y(N-1)
\end{array}\right] \\
& \bar{Y}_{c}=\left[\begin{array}{llll}
C \bar{B} & C \bar{A} \bar{B} & \cdots & C \bar{A}_{c}^{p-1} \bar{B}
\end{array}\right] \\
& V=\left[\begin{array}{ccccccc}
z(0) & z(1) & \cdots & z(p-1) & z(p) & \cdots & z(N-2) \\
& z(0) & \cdots & z(p-2) & z(p-1) & \cdots & z(N-3) \\
& & \ddots & \vdots & \vdots & & \vdots \\
& & & z(0) & z(1) & \cdots & z(N-p-1)
\end{array}\right]
\end{aligned}
$$

From Eq. (11) the set of observer Markov parameters of the closed-loop system given in Eq. (3) can be solved for provided that the additive excitation is sufficiently rich such that the $G v(i)$ rows in $V$ have full rank. The least-squares solution to the observer Markov parameter matrix $\bar{Y}_{c}$ is given as

$$
\overline{Y_{c}}=y V^{+}
$$

where $(.)^{+}$denotes the pseudo-inverse of the quantity in the parentheses.

For non-zero initial conditions a somewhat different equations must be used. The appropriate replacement equation for Eq. (11) is simply 


$$
y_{t}=\bar{Y}_{c} V_{t}
$$

where the truncated output matrix $y_{t}$ and the truncated input matrix $V_{1}$ are given as

$$
\begin{gathered}
y_{t}=\left[\begin{array}{llll}
y(p) & y(p+1) & \cdots & y(N-1)
\end{array}\right] \\
V_{t}=\left[\begin{array}{cccc}
z(p-1) & z(p) & \cdots & z(N-2) \\
z(p-2) & z(p-1) & \cdots & z(N-3) \\
\vdots & \vdots & & \vdots \\
z(0) & z(1) & \cdots & z(N-p-1)
\end{array}\right]
\end{gathered}
$$

and the solution for $\bar{Y}_{c}$ is

$$
\overline{Y_{c}}=y_{t} V_{t}^{+}
$$

The quantities $y_{t}$ and $V_{t}$ are obtained from $y$ and $V$ by simply deleting their first $p-1$ columns, respectively. This makes use of the fact that $\bar{A}^{i} \equiv 0$ for $i \geq p$, and the effect of the non-zero initial conditions can be neglected after $p$ times steps.

Similar to the analysis in Ref. 4, the number of observer Markov parameters characterized by the integer $p$, that are to be identified must be chosen such that $p q \geq n$, where $n$ is the order of the system and $q$ is the number of outputs. If $p$ observer Markov parameters are identified, then the maximum order of a system that can be realized is $p q$. Next, algebraic relations between the identified Markov parameters of the observer and those of the closed-loop system are described in the following.

\section{Relations Between Observer Markov Parameters and Closed-Loop System Markov Parameters}

Using the materials developed in the previous section, the observer Markov parameters of the closed-loop system can be computed from closed-loop data. This section shows that from the observer Markov parameters, the Markov parameters of the closed loop system can be recovered. Define $\bar{Y}_{c}(i)=\left[\begin{array}{ll}\bar{Y}_{c}^{(1)}(i) & \bar{Y}_{c}^{(2)}(i)\end{array}\right]$, where $\bar{Y}_{c}^{(1)}(i)$ and $\bar{Y}_{c}^{(2)}(i)$ are of dimensions $q \times m$ and $m \times m$, respectively. The Markov parameters of the closed loop system defined as 


$$
\begin{aligned}
Y_{c}(i) & =C A_{c}^{i-1} B \\
& =C(A+B F C)^{i-1} B
\end{aligned}
$$

can be recovered from the observer Markov parameters $\bar{Y}_{c}(i)$ according to the following relation

$$
Y_{c}(i)=\bar{Y}_{c}^{(1)}(i)+\sum_{i=1}^{i-1} \bar{Y}_{c}^{(2)}(\tau) Y_{c}(i-\tau)
$$

where $\bar{Y}_{c}(i) \equiv 0$ for $i \geq p$. In matrix form, the above recursive equation can be written as

$$
\left[\begin{array}{ccccc}
I & & & & \\
\bar{Y}_{c}^{(2)}(1) & I & & & \\
\bar{Y}_{c}^{(2)}(2) & \bar{Y}_{c}^{(2)}(1) & I & & \\
\vdots & \vdots & \ddots & \ddots & \\
\bar{Y}_{c}^{(2)}(i-1) & \bar{Y}_{c}^{(2)}(i-2) & \cdots & \bar{Y}_{c}^{(2)}(1) & I
\end{array}\right]\left[\begin{array}{c}
Y_{c}(1) \\
Y_{c}(2) \\
Y_{c}(3) \\
\vdots \\
Y_{c}(i)
\end{array}\right]=\left[\begin{array}{c}
\bar{Y}_{c}^{(1)}(1) \\
\bar{Y}_{c}^{(1)}(2) \\
\bar{Y}_{c}^{(1)}(3) \\
\vdots \\
\bar{Y}_{c}^{(1)}(i)
\end{array}\right]
$$

The matrix on the left hand side of Eq. (22) is square and full rank, thus for a given set of observer Markov parameters $\bar{Y}_{c}(i)$, the closed-loop system Markov parameters $Y_{c}(i)$ can be uniquely determined. The derivation of this convolution relation between the Markov parameters of the closed-loop system and those of its observer is analogous to the development presented in observer identification of Refs. 3-5, hence its proof is omitted.

\section{Relations Between Open-Loop and Closed-Loop System Markov Parameters with a Linear Output Feedback Controller}

Recall that the primary purpose of the problem is to compute the Markov parameters of the openIoop system. Sections I showed that from closed-loop data, the observer Markov parameters of the closed-loop system can be computed. Section II showed that from the observer Markov parameters, the Markov parameters of the closed-loop system can be found. This section will show that the Markov parameters of the open-loop system can be recovered from knowledge of the Markov parameters of the closed-loop system and the controller gain.

First, the first Markov parameter of the closed-loop system is the same as the first Markov parameter of the open-loop system, i.e., 


$$
Y(1)=Y_{c}(1)=C B
$$

Next, consider the closed-loop observer Markov parameter $Y_{c}(2)$

$$
\begin{aligned}
Y_{c}(2) & =C(A+B F C) B \\
& =C A B+C B F C B \\
& =Y(2)+Y_{c}(1) F Y(1)
\end{aligned}
$$

from which the open-loop system Markov parameter $Y(2)$ is simply,

$$
Y(2)=Y_{c}(2)-Y_{c}(1) F Y(1)
$$

Similarly, the open-loop system Markov parameter $Y(3)$ can be recovered by considering the closed-loop Markov parameter $Y_{c}(3)$,

$$
\begin{aligned}
Y_{c}(3) & =C(A+B F C)^{2} B \\
& =C A^{2} B+C B F C A B+C(A+B F C) B F C B \\
& =Y(3)+Y_{c}(1) F Y(2)+Y_{c}(2) F Y(1)
\end{aligned}
$$

which yields

$$
Y(3)=Y_{c}(3)-Y_{c}(1) F Y(2)-Y_{c}(2) F Y(1)
$$

By induction, the relationship between the Markov parameters of the closed-loop system given in Eq. (3) and those of the open-loop system given in Eq. (1) is a weighted convolution sum, with the weighting matrix being the closed-loop gain $F$. In general, this expression is written as

$$
Y(i)=Y_{c}(i)-\sum_{\tau=1}^{i-1} Y_{c}(\tau) F Y(i-\tau)
$$

for $i=1,2,3, \ldots$. The above recursive equation can be written in matrix form as 


$$
\left[\begin{array}{ccccc}
I & & & & \\
Y_{c}(1) F & I & & & \\
Y_{c}(2) K & Y_{c}(1) F & I & & \\
\vdots & \vdots & \ddots & \ddots & \\
Y_{c}(i-1) K & Y_{c}(i-2) K & \ldots & Y_{c}(1) F & I
\end{array}\right]\left[\begin{array}{c}
Y(1) \\
Y(2) \\
Y(3) \\
\vdots \\
Y(i)
\end{array}\right]=\left[\begin{array}{c}
Y_{c}(1) \\
Y_{c}(2) \\
Y_{c}(3) \\
\vdots \\
Y_{c}(i)
\end{array}\right]
$$

Each product $Y_{c}(i) F$ is a $q \times q$ square matrix. The left-hand side matrix in Eq. (29) is square and full rank. This implies that the open-loop system Markov parameters can be uniquely recovered from the closed-loop system Markov parameters by this method.

\section{Closed-Loop Identification of System with Direct Transmission Term}

In the basic problem considered in Sections I-III, the system description does not contain a direct transmission term. In identification and control of flexible structures, accelerometers are often used as measurement sensors, which will introduce a direct transmission term in the state space model. Identification of such systems in the closed-loop is slightly more complicated. Consider a state space model with a direct transmission term $D$,

$$
\begin{aligned}
x(i+1) & =A x(i)+B u(i) \\
y(i) & =C x(i)+D u(i)
\end{aligned}
$$

where again $x(i) \in R^{n}, y(i) \in R^{q}, u(i) \in R^{m}$. The input equation given in Eq. (2) in this case is

$$
\begin{aligned}
u(i) & =F y(i)+G v(i) \\
& =F C x(i)+F D u(i)+G v(i)
\end{aligned}
$$

The closed-loop system dynamics can be derived as follows. First, from Eq. (31), the controller input can be expressed as

$$
u(i)=(I-F D)^{-1} F C x(i)+(I-F D)^{-1} G v(i)
$$

provided that the inverse $(I-F D)^{-1}$ exists. Using Eq. (32) in Eq. (30) yields the following set of closed-loop dynamics equations describing the relationship between the excitation $G v(i)$ and output $y(i)$ 


$$
\begin{aligned}
x(i+1) & =\left[A+B(I-F D)^{-1} F C\right] x(i)+B(I-F D)^{-1} G v(i) \\
y(i) & =(I-D F)^{-1} C x(i)+(I-D F)^{-1} D G v(i)
\end{aligned}
$$

provided the inverse $(I-D F)^{-1}$ exists. To derive the observer equations for the above system, add and subtract $M y(i)$ to the right hand side of the state equation in Eq. (33)

$$
\begin{aligned}
x(i+1) & =A_{c} x(i)+B_{c} G v(i)+M y(i)-M y(i) \\
& =A_{c} x(i)+B_{c} G v(i)+M[C x(i)+D u(i)]-M y(i) \\
& =\left(A_{c}+M C\right) x(i)+M D u(i)+B_{c} G v(i)-M y(i) \\
& =\left(A_{c}+M C\right) x(i)+M D[F y(i)+G v(i)]+B_{c} G v(i)-M y(i) \\
& =\left(A_{c}+M C\right) x(i)+\left(B_{c}+M D\right) G v(i)-M(I-D F) y(i)
\end{aligned}
$$

For simplicity of notation, the set of observer equations to be used for identification is

$$
\begin{aligned}
x(i+1) & =\bar{A}_{c} x(i)+\bar{B}_{c} z(i) \\
y(i) & =C x(i)+D u(i)
\end{aligned}
$$

where

$$
\begin{array}{ccc}
\bar{A}_{c}=A_{c}+M C, & A_{c}=A+B_{c} F C, & B_{c}=B(I-F D)^{-1} \\
\bar{B}_{c}=\left[\begin{array}{lll}
B_{c}+M D & -M(I-D F)
\end{array}\right], & z(i)=\left[\begin{array}{c}
G v(i) \\
y(i)
\end{array}\right]
\end{array}
$$

The input-output description of the above system with zero initial conditions is

$$
y(i)=\sum_{\tau=0}^{i-1} C \bar{A}_{c}^{i-\tau-1} \bar{B}_{c} z(\tau)+D u(0)
$$

Let $M$ be a deadbeat observer gain, then the input-output relation can be expressed as

$$
y(i)=\sum_{k=1}^{p} \bar{Y}_{c}(\tau) z(i-\tau)+D u(i)
$$

Writing in matrix form, the closed-loop observer Markov parameters are related to set of inputoutput data by the following equation 


$$
y=\bar{Y}_{c} V
$$

where

$$
\begin{aligned}
& y=\left[\begin{array}{llllllll}
y(0) & y(1) & y(2) & \cdots & y(p) & y(p+1) & \cdots & y(N-1)
\end{array}\right] \\
& \bar{Y}_{c}=\left[\begin{array}{lllll}
D & C \bar{B}_{c} & C \bar{A}_{c} \bar{B}_{c} & \cdots & C \bar{A}_{c}^{p-1} \bar{B}_{c}
\end{array}\right] \\
& V=\left[\begin{array}{cccccccc}
u(0) & u(1) & u(2) & \cdots & u(p) & u(p+1) & \cdots & u(N-1) \\
& z(0) & z(1) & \cdots & z(p-1) & z(p) & \cdots & z(N-2) \\
& & z(0) & \cdots & z(p-2) & z(p-1) & \cdots & z(N-3) \\
& & & \ddots & \vdots & \vdots & & \vdots \\
& & & & z(0) & z(1) & \cdots & z(N-p-1)
\end{array}\right]
\end{aligned}
$$

Equation (42) can be used to solve for the closed-loop observer Markov parameters. The solution is given in Eq. (15), with the exception that the output and input matrices are now given in Eq. (41) and Eq. (43), respectively. If the initial conditions are not zero, then the truncated version of the corresponding output and input matrices are

$$
\begin{gathered}
y_{t}=\left[\begin{array}{llll}
y(p) & y(p+1) & \cdots & y(N-1)
\end{array}\right] \\
V_{\iota}=\left[\begin{array}{cccc}
u(p) & u(p+1) & \cdots & u(N-1) \\
z(p-1) & z(p) & \cdots & z(N-2) \\
z(p-2) & z(p-1) & \cdots & z(N-3) \\
\vdots & \vdots & & \vdots \\
z(0) & z(1) & \cdots & z(N-p-1)
\end{array}\right]
\end{gathered}
$$

The observer parameter matrix is the same as in Eq. (42). Again, in order to be able to solve for the observer Markov parameter matrix $\bar{Y}_{c}$, the rows of $G v(i)$ in $z(i)$ must be full rank. Also, the number of observer Markov parameters characterized by the integer $p$, that are to be identified, must be chosen such that $p q \geq n$.

The next step is to show that the open-loop system Markov parameters 


$$
Y(i)=C A^{i-1} B, i=1,2, \ldots, p, p+1, \ldots
$$

can be recovered from the identified observer Markov parameters

$$
\bar{Y}_{c}(i)=C \bar{A}_{c}^{i-1} \bar{B}_{c}=\left[C \bar{A}_{c}^{i-1}\left(B_{c}+M D\right),-C \bar{A}_{c}^{i-1} M(I-D F)\right], \quad i=1,2, \ldots, p
$$

and

$$
\bar{Y}_{c}(i)=0, \quad i=p+1, p+2, \ldots
$$

Define the first and second partitions of $\bar{Y}_{c}(i)$ as

$$
\begin{aligned}
\bar{Y}_{c}^{(1)}(i) & =C \bar{A}_{c}^{i-1}\left(B_{c}+M D\right) \\
& =C\left[A+B(I-F D)^{-1} F C+M C\right]^{i-1}\left[B(I-F D)^{-1}+M D\right] \\
\bar{Y}_{c}^{(2)}(i) & =-C \bar{A}_{c}^{i-1} M(I-D F) \\
& =-C\left[A+B(I-F D)^{-1} F C+M C\right]^{i-1} M(I-D F)
\end{aligned}
$$

The direct transmission term is identified directly,

$$
D=\bar{Y}_{c}(0)
$$

From $\bar{Y}_{c}(i)$ the Markov parameters sequences $Y_{c}(i)=C A_{c}^{i-1} B_{c}, i=1,2, \ldots, p, p+1, \ldots$ can be computed from the following recursive equation

$$
Y_{c}(i)=\bar{Y}_{c}^{(1)}(i)+\sum_{\tau=1}^{i} \bar{Y}_{c}^{(2)}(\tau)(I-D F)^{-1} Y_{c}(i-\tau)
$$

where $Y_{c}(0)=D$ and $\bar{Y}_{c}(i)=0, \quad i=p+1, p+2, \ldots$ The Markov parameters $Y_{c}(i)$,

$$
Y_{c}(i)=C A_{c}^{i-1} B_{c}=C\left(A+B_{c} F C\right)^{i-1} B_{c}
$$

computed above, have the same structure as the Markov parameters $Y_{c}(i)$ given in Eq. (20) where $B_{c}=B(I-F D)^{-1}$ now plays the role of $B$. Therefore, the open-loop system Markov parameters $Y(i)=C A^{i-1} B$ can be computed from $Y_{c}(i)=C\left(A+B_{c} F C\right)^{i-1} B_{c}$ as 


$$
Y(i)=Y_{c}(i)(I-F D)-\sum_{i=1}^{i-1} Y_{c}(\tau) F Y(i-\tau)
$$

\section{Closed-loop Identification of System with a Dynamic Feedback Controller}

In this section, the identification of the open-loop model from a closed-loop system having an existing dynamic feedback controller is considered. Consider again a system in state space representation,

$$
\begin{aligned}
x(i+1) & =A x(i)+B u(i) \\
y(i) & =C x(i)+D u(i)
\end{aligned}
$$

where $x(i) \in R^{n}, y(i) \in R^{q}, u(i) \in R^{m}$. A dynamic feedback controller is used to control the system which for closed-loop identification is excited according to

$$
\begin{aligned}
s(i+1) & =P s(i)+Q w(i)+v_{2}(i) \\
g(i) & =R s(i)+S w(i)
\end{aligned}
$$

where $s(i) \in R^{n}, g(i) \in R^{m}, w(i) \in R^{q}$. The scalar $n_{s}$ denotes the order of the dynamic controller, which in general is less than or equal to the order of the open-loop system, $n_{s} \leq n$. The dynamic controller interacts with the system via

$$
\begin{aligned}
& y(i)=w(i) \\
& u(i)=g(i)+v_{1}(i)
\end{aligned}
$$

The vector quantities $v_{1}(i)$ and $v_{2}(i)$ denote the additive excitation signals for closed-loop identification. First, the closed-loop dynamics is derived. The input to the system can be expressed as

$$
\begin{aligned}
u(i) & =R s(i)+S w(i)+v_{1}(i) \\
& =R s(i)+S y(i)+v_{1}(i) \\
& =R s(i)+S[C x(i)+D u(i)]+v_{1}(i)
\end{aligned}
$$

from which the input $u(i)$ can be expressed as 


$$
u(i)=(I-S D)^{-1}\left[R . s(i)+S C x(i)+v_{1}(i)\right]
$$

provided that the inverse $(I-S D)^{-1}$ exists. Substituting Eq. (59) into the state equation in Eq. (55), and the controller state equation in Eq. (56) yields

$$
\begin{aligned}
x(i+1) & =A x(i)+B(I-S D)^{-1}\left[R s(i)+S C x(i)+v_{1}(i)\right] \\
& =\left[A+B(I-S D)^{-1} S C\right] x(i)+\left[B(I-S D)^{-1} R\right] s(i)+\left[B(I-S D)^{-1}\right] v_{1}(i)
\end{aligned}
$$

and

$$
\begin{aligned}
s(i+1) & =P s(i)+Q[C x(i)+D u(i)]+v_{2}(i) \\
& =P s(i)+Q C x(i)+Q D(I-S D)^{-1}\left[R s(i)+S C x(i)+v_{1}(i)\right]+v_{2}(i) \\
& =\left[Q C+Q D(I-S D)^{-1} S C\right] x(i)+\left[P+Q D(I-S D)^{-1} R\right] s(i)+\left[Q D(I-S D)^{-1}\right] v_{1}(i)+v_{2}(i)
\end{aligned}
$$

Equations (60) and (61) can be combined in matrix form as

$$
\left[\begin{array}{l}
x(i+1) \\
s(i+1)
\end{array}\right]=\left[\begin{array}{cc}
A+B(I-S D)^{-1} S C & B(I-S D)^{-1} R \\
Q C+Q D(I-S D)^{-1} S C & P+Q D(I-S D)^{-1} R
\end{array}\right]\left[\begin{array}{c}
x(i) \\
S(i)
\end{array}\right]+\left[\begin{array}{cc}
B(I-S D)^{-1} & 0 \\
Q D(I-S D)^{-1} & I
\end{array}\right]\left[\begin{array}{l}
v_{1}(i) \\
v_{2}(i)
\end{array}\right]
$$

Recall that

$$
\begin{gathered}
u(i)=R s(i)+S y(i)+v_{1}(i) \\
s(i+1)=P s(i)+Q y(i)+v_{2}(i)
\end{gathered}
$$

One can write Eq. (63) and Eq. (64) together in a matrix form as

$$
\left[\begin{array}{c}
u(i) \\
s(i+1)
\end{array}\right]=\left[\begin{array}{ll}
S & R \\
Q & P
\end{array}\right]\left[\begin{array}{l}
y(i) \\
s(i)
\end{array}\right]+\left[\begin{array}{c}
v_{1}(i) \\
v_{2}(i)
\end{array}\right]
$$

Furthermore, the system output equation and the controller state equation can be combined into a single matrix equation,

$$
\left[\begin{array}{l}
y(i) \\
s(i)
\end{array}\right]=\left[\begin{array}{ll}
C & 0 \\
0 & I_{n}
\end{array}\right]\left[\begin{array}{l}
x(i) \\
s(i)
\end{array}\right]+\left[\begin{array}{cc}
D & 0 \\
0 & 0
\end{array}\right]\left[\begin{array}{c}
u(i) \\
s(i+1)
\end{array}\right]
$$


In the following, a procedure is developed to solve for the Markov parameters of the open-loop system from the Markov parameters of the closed-loop system when the dynamics controller is known. Define the following augmented system characterized by the matrices 9

$$
A_{a}=\left[\begin{array}{ll}
A & 0 \\
0 & 0
\end{array}\right], \quad B_{a}=\left[\begin{array}{cc}
B & 0 \\
0 & I_{n}
\end{array}\right], \quad C_{a}=\left[\begin{array}{ll}
C & 0 \\
0 & I_{m}
\end{array}\right], \quad D_{a}=\left[\begin{array}{ll}
D & 0 \\
0 & 0
\end{array}\right], \quad F_{a}=\left[\begin{array}{ll}
S & R \\
Q & P
\end{array}\right]
$$

and the following augmented state, input, output, and excitation vectors

$$
x_{a}(i)=\left[\begin{array}{l}
x(i) \\
s(i)
\end{array}\right], \quad u_{a}(i)=\left[\begin{array}{c}
u(i) \\
s(i+1)
\end{array}\right], \quad y_{a}(i)=\left[\begin{array}{l}
y(i) \\
s(i)
\end{array}\right], \quad v_{a}(i)=\left[\begin{array}{c}
v_{1}(i) \\
v_{2}(i)
\end{array}\right]
$$

Making use of the definitions above, Eq. (65) and Eq. (66) become, respectively,

$$
\begin{gathered}
u_{a}(i)=F_{a} y_{a}(i)+v_{a}(i) \\
y_{a}(i)=C_{a} x_{a}(i)+D_{a} u_{a}(i)
\end{gathered}
$$

Furthermore, define a matrix $X_{a}$ to be

$$
X_{a}=\left[\begin{array}{cc}
(I-S D)^{-1} & 0 \\
Q D(I-S D)^{-1} & I
\end{array}\right]
$$

It can be verified by direct substitution that the following identities hold

$$
\begin{gathered}
{\left[\begin{array}{cc}
A+B(I-S D)^{-1} S C & B(I-S D)^{-1} R \\
Q C+Q D(I-S D)^{-1} S C & P+Q D(I-S D)^{-1} R
\end{array}\right]=A_{a}+B_{a} X_{a} F_{a} C_{a}} \\
{\left[\begin{array}{cc}
B(I-S D)^{-1} & 0 \\
Q D(I-S D)^{-1} & I
\end{array}\right]=B_{a} X_{a}} \\
X_{a}=\left[\begin{array}{cc}
I-D S & 0 \\
-Q D & I
\end{array}\right]^{-1}=\left(I-F_{a} D_{a}\right)^{-1}
\end{gathered}
$$


Using these relations, the set of equations describing closed-loop dynamics becomes

$$
\begin{aligned}
x_{a}(i+1) & =\left[A_{a}+B_{a}\left(I-F_{a} D_{a}\right)^{-1} F_{a} C_{a}\right] x_{a}(i)+B_{a}\left(I-F_{a} D_{a}\right)^{-1} v_{a}(i) \\
y_{a}(i) & =\left(I-D_{a} F_{a}\right)^{-1} C_{a} x_{a}(i)+\left(I-D_{a} F_{a}\right)^{-1} D_{a} v_{a}(i)
\end{aligned}
$$

where the first equation in Eq. (75) is obtained by direct use of the identities in Eqs. (67) in Eq. (62), and the second equation is obtained by substituting Eq. (69) into Eq. (70) and solving for $y_{a}(i)$ provided that the inverse $\left(I-D_{a} F_{a}\right)^{-1}$ exists. As before, the additive excitation does not affect the overall system stability that is provided by the dynamic feedback controller. Comparing Eq. (75) to Eq. (33) reveals that the augmented system for the closed-loop dynamic controller has the same form as the following system

$$
\begin{array}{r}
x_{a}(i+1)=A_{a} x_{a}(i)+B_{a} u_{a}(i) \\
y_{a}(i)=C_{a} x_{a}(i)+D_{a} u_{a}(i)
\end{array}
$$

with an output feedback law for $u_{a}(i)$ given in Eq. (69), that includes an excitation term $v_{a}(i)$. The observer equations for the augmented system can be derived by adding and subtracting the term $M_{a} y_{a}(i)$ to the state equation in Eq. (75),

$$
\begin{aligned}
x_{a}(i+1) & =A_{a c} x_{a}(i)+B_{a c} \nu_{a}(i)+M_{a} y_{a}(i)-M_{a} y_{a}(i) \\
& =A_{a c} x_{a}(i)+B_{a c} v_{a}(i)+M_{a}\left[C_{a} x_{a}(i)+D_{a} u_{a}(i)\right]-M_{a} y_{a}(i) \\
& =\left(A_{a c}+M_{a} C_{a}\right) x_{a}(i)+M_{a} D_{a}\left[F_{a} y_{a}(i)+v_{a}(i)\right]+B_{a c} v_{a}(i)-M_{a} y_{a}(i) \\
& =\left(A_{a c}+M_{a} C_{a}\right) x_{a}(i)+\left(B_{a c}+M_{a} D_{a}\right) v_{a}(i)-M_{a}\left(I-D_{a} F_{a}\right) y_{a}(i)
\end{aligned}
$$

The set of observer equations to he used for identification is simply

$$
\begin{aligned}
x_{a}(i+1) & =\bar{A}_{a} x_{a}(i)+\bar{B}_{a} z(i) \\
y_{a}(i) & =C_{a} x_{a}(i)+D_{a} u_{a}(i)
\end{aligned}
$$

where

$$
\bar{A}_{a c}=A_{a r}+M_{a} C_{a}, \quad A_{a c}=A_{a}+B_{a c} F_{a} C_{a}, \quad B_{a c}=B_{a}\left(I-F_{a} D_{a}\right)^{-1}
$$




$$
\bar{B}_{\alpha c}=\left[\begin{array}{ll}
B_{\alpha c}+M_{a} D_{a} & -M_{a}\left(I-D_{a} F_{a}\right)
\end{array}\right], \quad z_{a}(i)=\left[\begin{array}{l}
v_{a}(i) \\
y_{a}(i)
\end{array}\right]
$$

The observer Markov parameters for the system given in Eq. (75) are

$$
\begin{gathered}
\bar{Y}_{a c}(0)=D_{a} \\
\bar{Y}_{a c}(i)=C_{a} \bar{A}_{a c}^{i-1} \bar{B}_{\alpha c}=\left[C_{a} \bar{A}_{a c}^{i-1}\left(B_{a c}+M_{a} D_{a}\right),-C_{a} \bar{A}_{a c}^{i-1} M_{a}\left(I-D_{a} F_{a}\right)\right], \quad i=1,2, \ldots, p
\end{gathered}
$$

Assuming zero initial conditions, the observer Markov parameters can be solved from

$$
y_{a}=\bar{Y}_{a c} V_{a}
$$

where

$$
\begin{gathered}
y_{a}=\left[\begin{array}{llllllll}
y_{a}(0) & y_{a}(1) & y_{a}(2) & \cdots & y_{a}(p) & y_{a}(p+1) & \cdots & y_{a}(N-1)
\end{array}\right] \\
\bar{Y}_{a c}=\left[\begin{array}{llllllll}
D & D_{a} & C_{a} \bar{B}_{a c} & C_{a} \bar{A}_{a c} \bar{B}_{a c} & \cdots & C_{a} \bar{A}_{a c}^{p-1} \bar{B}_{a c}
\end{array}\right] \\
V_{a}=\left[\begin{array}{cccccccc}
u_{a}(0) & u_{a}(1) & u_{a}(2) & \cdots & u_{a}(p) & u_{a}(p+1) & \cdots & u_{a}(N-1) \\
& z_{a}(0) & z_{a}(1) & \cdots & z_{a}(p-1) & z_{a}(p) & \cdots & z_{a}(N-2) \\
& & z_{a}(0) & \cdots & z_{a}(p-2) & z_{a}(p-1) & \cdots & z_{a}(N-3) \\
& & & \ddots & \vdots & \vdots & & \vdots \\
& & & z_{a}(0) & z_{a}(1) & \cdots & z_{a}(N-p-1)
\end{array}\right]
\end{gathered}
$$

If the initial conditions are not zero, then the truncated versions of $y_{a}$ and $V_{a}$ are to be used. They are obtained by simply deleting the first $p$ columns of $y_{a}$ and $V_{a}$, respectively. In this case, the number of observer Markov parameters characterized by the integer $p$, that are to be identified must be chosen such that $p\left(q+n_{s}\right) \geq n+n_{s}$.

As before, define the first and second partitions of $\bar{Y}_{a c}(i)$ as

$$
\begin{aligned}
\bar{Y}_{a c}^{(1)}(i) & =C_{a} \bar{A}_{a c}^{i-1}\left(B_{a c}+M_{a} D_{a}\right) \\
& =C_{a}\left[A_{a}+B_{a}\left(I-F_{a} D_{a}\right)^{-1} F_{a} C_{a}+M_{a} C_{a}\right]^{i-1}\left[B_{a}\left(I-F_{a} D_{a}\right)^{-1}+M_{a} D_{a}\right]
\end{aligned}
$$




$$
\begin{aligned}
\bar{Y}_{a c}^{(2)}(i) & =-C_{a} \bar{A}_{a c}^{i-1} M_{a}\left(I-D_{a} F_{a}\right) \\
& =-C_{a}\left[A_{a}+B_{a}\left(I-F_{a} D_{a}\right)^{-1} F_{a} C_{a}+M_{a} C_{a}\right]^{i-1} M_{a}\left(I-D_{a} F_{a}\right)
\end{aligned}
$$

The recursive equations for the Markov parameters for $Y_{a r}(i)=C_{a} A_{a c}^{i-1} B_{a}$ and $Y_{a}(i)=C_{a} A_{a}^{i-1} B_{a}$ for $i=1,2, \ldots, p, p+1, \ldots$ are

$$
\begin{gathered}
Y_{a c}(i)=\bar{Y}_{a c}^{(1)}(i)+\sum_{\tau=1}^{i} \bar{Y}_{a c}^{(2)}(\tau)\left(I-D_{a} F_{a}\right)^{-1} Y_{a c}(i-\tau) \\
Y_{a}(i)=Y_{a c}(i)\left(I-F_{a} D_{a}\right)-\sum_{\tau=1}^{i-1} Y_{a c}(\tau) F_{a} Y_{a}(i-\tau)
\end{gathered}
$$

where $\bar{Y}_{a c}(0)=Y_{a c}(0)=D_{a}$, and $\bar{Y}_{a c}(i)=0$ for $i=p+1, p+2, \ldots$ In this procedure, the Markov parameters $Y_{\alpha c}(i)$ are first computed from the identified observer Markov parameters $\bar{Y}_{\alpha c}(i)$ by using Eq. (89). Then, the Markov parameters $Y_{a}(i)$ are computed from the Markov parameters $Y_{a c}(i)$ by using Eq. (90)). Finally, it remains to be shown that the open-loop system Markov parameters $Y(i)$ can be recovered from the Markov parameters $Y_{a}(i)$. This is simple, since

$$
Y_{a}(0)=\left[\begin{array}{cc}
Y(0) & 0 \\
0 & 0
\end{array}\right], \quad Y_{a}(1)=\left[\begin{array}{cc}
Y(1) & 0 \\
0 & 1
\end{array}\right], \quad Y_{a}(i)=\left[\begin{array}{cc}
Y(i) & 0 \\
0 & 0
\end{array}\right], i=2,3, \ldots
$$

Thus, the open-loop system Markov parameters $Y(0)=D, Y(i)=C A^{i-1} B, i=1,2, \ldots$ are the upper left partitions of the computed Markov parameters $Y_{a}(i), i=0,1,2, \ldots$

\section{v. Identification from Combined Markov Parameters}

In practice, the open-loop system considered in previous sections may actually include the plan: and the input/output filters. If the dynamics of the open-loop system is known, and the filter dynamics is known, then under certain conditions, it is possible to recover the dynamics of the plant alone. Mathematically, this is the case of a combined system consisting of two cascading linear systems, and the problem is to compute the Markov parameters of one system if the Markov parameters of the other system and those of the combined system are known. First, the relationship between the Markov parameters of the combined system and those of the individual systems need to be derived. Let one system denoted by 


$$
\begin{array}{r}
x_{1}(i+1)=A_{1} x_{1}(i)+B_{1} u_{1}(i) \\
y_{1}(i)=C_{1} x_{1}(i)+D_{1} u_{1}(i)
\end{array}
$$

be preceded by another system

$$
\begin{aligned}
x_{2}(i+1) & =A_{2} x_{2}(i)+B_{2} u_{2}(i) \\
y_{2}(i) & =C_{2} x_{2}(i)+D_{2} u_{2}(i)
\end{aligned}
$$

where $x_{1}(i) \in R^{n_{1}}, y_{1}(i) \in R^{q_{1}}, u_{1}(i) \in R^{m_{1}}$, and $x_{2}(i) \in R^{n_{2}}, y_{2}(i) \in R^{q_{2}}, u_{2}(i) \in R^{m_{2}}$. Let the output of the second system be the input to the first system, i.e., $q_{2}=m_{1}$, and

$$
y_{2}(i)=u_{1}(i)
$$

Then the combined system dynamics is given as

$$
\begin{aligned}
x_{t}(i+1) & =A_{1} x_{t}(i)+B_{1} u_{2}(i) \\
y_{1}(i) & =C_{1} x_{1}(i)+D_{1} u_{2}(i)
\end{aligned}
$$

where

$$
A_{t}=\left[\begin{array}{cc}
A_{1} & B_{1} C_{2} \\
0 & A_{2}
\end{array}\right] \quad B_{1}=\left[\begin{array}{c}
B_{1} D_{2} \\
B_{2}
\end{array}\right] \quad C_{1}=\left[\begin{array}{lll}
C_{1} & D_{1} C_{2}
\end{array}\right] \quad D_{1}=D_{1} D_{2} \quad x_{1}(i)=\left[\begin{array}{l}
x_{1}(i) \\
x_{2}(i)
\end{array}\right]
$$

The relationship between the Markov parameters of the combined system, and the Markov parameters of the individual systems is established in the following.

First,

$$
Y_{t}(0)=D_{t}=D_{1} D_{2}=Y_{1}(0) Y_{2}(0)
$$

Next,

$$
\begin{aligned}
Y_{\imath}(1) & =C_{1} B_{1}=C_{1} B_{1} D_{2}+D_{1} C_{2} B_{2} \\
& =Y_{1}(1) Y_{2}(0)+Y_{1}(0) Y_{2}(1)
\end{aligned}
$$


Similarly,

$$
\begin{gathered}
Y_{1}(2)=C_{1} A_{1} B_{t}=C_{1} A_{1} B_{1} D_{2}+C_{1} B_{1} C_{2} B_{2}+D_{1} C_{2} A_{2} B_{2} \\
=Y_{1}(2) Y_{2}(0)+Y_{1}(1) Y_{2}(1)+Y_{1}(0) Y_{2}(2)
\end{gathered}
$$

By induction, the general relationship is given as

$$
Y_{1}(i)=\sum_{\tau=0}^{i} Y_{1}(\tau) Y_{2}(i-\tau)
$$

Using this general relationship, under certain conditions, it is possible to solve for the Markov parameters of one system when the Markov parameters of the combined system and of the other system are known. In particular, the following cases apply.

If the Markov parameters of the combined system and of system 1 are known, then the Markov parameters of system 2 can be solved using the following equations.

$$
\begin{gathered}
Y_{2}(0)=\left[Y_{1}^{T}(0) Y_{1}(0)\right]^{-1} Y_{1}^{T}(0) Y_{1}(0) \\
Y_{2}(i)=\left[Y_{1}^{T}(0) Y_{1}(0)\right]^{-1} Y_{1}^{T}(0)\left[Y_{\imath}(i)-\sum_{\tau=0}^{i-1} Y_{1}(\tau) Y_{2}(i-\tau)\right], \quad i=1,2, \ldots
\end{gathered}
$$

provided $q_{1} \geq m_{1}$, and $Y_{1}(0)=D_{1}$ has full (column) rank.

On the other hand, if the Markov parameters of the combined system and of system 2 are known, then the Markov parameters of system 1 can be solved according to the following equations.

$$
\begin{gathered}
Y_{1}(0)=Y_{1}(0) Y_{2}^{T}(0)\left[Y_{2}(0) Y_{2}^{T}(0)\right]^{-1} \\
Y_{2}(i)=\left[Y_{\imath}(i)-\sum_{\tau=0}^{i-1} Y_{1}(\tau) Y_{2}(i-\tau)\right] Y_{2}^{T}(0)\left[Y_{2}(0) Y_{2}^{T}(0)\right]^{-1}, \quad i=1,2, \ldots
\end{gathered}
$$


provided $m_{2} \geq q_{2}$, and $Y_{2}(0)=D_{2}$ has full (row) rank.

\section{State Space Realization by ERA}

From the recovered open-loop Markov parameters, a state space model of the system can be obtained by the Eigensystem Realization Algorithm, which is outlined in this section. The algorithm begins with an $r \times s$ block data matrix, denoted by $H(i-1)$

$$
H(i-1)=\left[\begin{array}{cccc}
Y(i) & Y(i+1) & \cdots & Y(i+s-1) \\
Y(i+1) & Y(i+2) & \cdots & Y(i+s) \\
\vdots & \vdots & \cdots & \vdots \\
Y(i+r-1) & Y(i+r) & \cdots & Y(i+r+s-2)
\end{array}\right]
$$

where $Y(i)=C A^{i-1} B, i=1,2, \ldots$ The order of the system is determined from the singular value decomposition of $\boldsymbol{H ( 0 )}$,

$$
H(0)=U \Sigma V^{T}=U_{1} S_{1} V_{1}^{T}
$$

where the columns of $U_{1}$ and $V_{1}$ are orthonormal, $S_{1}$ is an $n \times n$ diagonal matrix of retained positive singular values, and $n$ is the order of the system. Defining a $q \times r q$ matrix $E_{q}^{T}$, and an $m \times s m$ matrix $E_{m}^{T}$ made up of identity and null matrices of the form

$$
E_{q}^{r}=\left[\begin{array}{ll}
I_{q \times q} & O_{q \times(r-1) q}
\end{array}\right], \quad E_{m}^{r}=\left[\begin{array}{ll}
I_{m \times m} & O_{m \times(s-1) m}
\end{array}\right]
$$

A discrete-time minimal order realization of the system can be shown to be

$$
\begin{aligned}
& A_{r}=S_{1}^{-1 / 2} U_{1}^{T} H(1) V_{1} S_{1}^{-1 / 2} \\
& B_{r}=S_{1}^{1 / 2} V_{1}^{T} E_{m} \\
& C_{r}=E_{q}^{T} U_{1} S_{1}^{1 / 2}
\end{aligned}
$$

This is the basic ERA formulation. To use ERA in the present identification procedure, the entries that make up the data matrix given in Eq. (106) are precisely the recovered system Markov 
parameters $Y(i)=C A^{i-1} B, i=1,2, \ldots$ For further details on the algorithm, the readers are referred to various references in the literature, e.g., Refs. 10, 11.

\section{Numerical Examples}

In this section, several examples are provided to illustrate the identification procedure for closedloop system developed in this paper. Example 1 illustrates the simple case of identifying a system with an existing linear output feedback controller. The case of closed-loop identification of a system with a direct transmission term is shown in Example 2. Example 3 presents a case with a dynamic feedback controller. The system matrices are given here so that the readers can reproduce the results. It is the purpose of the following examples to illustrate step-by-step the series of computations involved for each of the respective closed-loop case, not the controllers used.

Example 1: Consider a two-input three-output three degree-of-freedom (sixth-order) mass, spring, dashpot system whose discrete system matrices are given below.

$$
\begin{gathered}
A=\left[\begin{array}{ccccccc}
0.9691 & 0.0154 & 0.0001 & 0.2120 & 0.0014 & 0.0000 \\
0.0154 & 0.9690 & 0.0155 & 0.0014 & 0.2139 & 0.0014 \\
0.0000 & 0.0077 & 0.9768 & 0.0000 & 0.0007 & 0.2127 \\
-0.2817 & 0.1395 & 0.0009 & 0.9458 & 0.0180 & 0.0001 \\
0.1407 & -0.2838 & 0.1412 & 0.0180 & 0.9634 & 0.0182 \\
0.0005 & 0.0699 & -0.2122 & 0.00 \times 10 & 0.0091 & 0.9544
\end{array}\right] \quad B=\left[\begin{array}{lll}
0.0232 & 0.0001 \\
0.0001 & 0.0233 \\
0.0000 & 0.0000 \\
0.2120 & 0.0014 \\
0.0014 & 0.2139 \\
0.0000 & 0.0007
\end{array}\right] \\
C=\left[\begin{array}{llllll}
0 & 0 & 0 & 1 & 0 & 0 \\
0 & 0 & 0 & 0 & 1 & 0 \\
0 & 0 & 0 & 0 & 0 & 1
\end{array}\right] \quad D=\left[\begin{array}{ll}
0 & 0 \\
0 & 0 \\
0 & 0
\end{array}\right]
\end{gathered}
$$

The inputs are the applied forces at two of the masses, and the outputs are the three velocity measurements. Let the system be stabilized by a linear feedback controller of the form

$$
u(i)=F y(i)
$$

where the feedback gain is given as 


$$
F=\left[\begin{array}{ccc}
-0.375 & 0 & 0 \\
0 & -0.500 & 0
\end{array}\right]
$$

For closed-loop identification, the closed-loop be excited by a random perturbation of the system output $y(i)$, i.e.,

$$
u(i)=F[y(i)+v(i)]
$$

In this case, $G=F$ in Eq. (2). The excitation signal for the second output is shown in Fig. 2, and the resultant closed-loop system response for the second output is shown in Fig. 3.

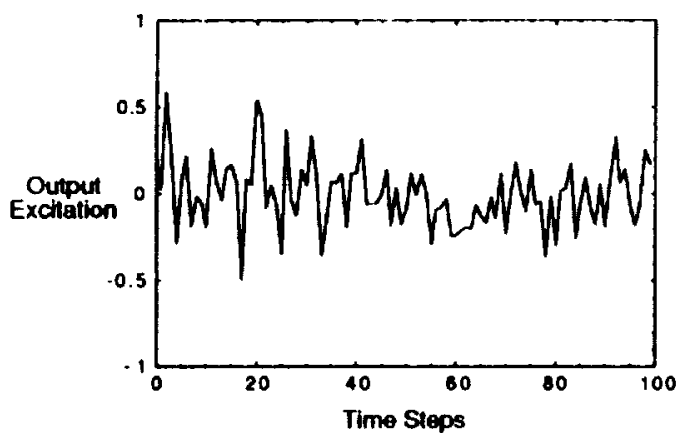

Fig. 2: Excitation signal for closed-loop system by output perturbation.

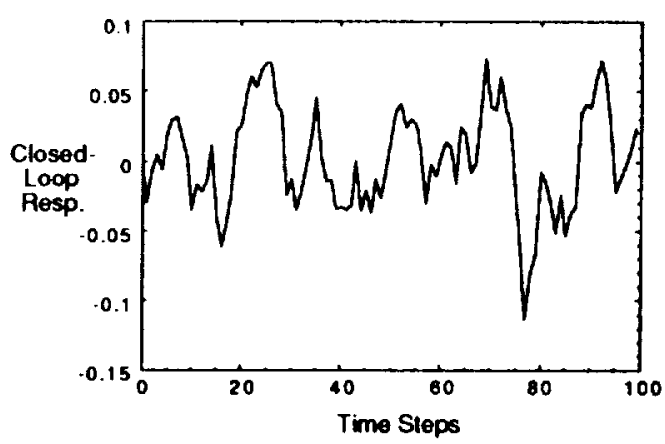

Fig. 3: Response of closed-loop system to excitation signal.

Using data from the excitation, the observer Markov parameters for the closed-loop system are identified. Since the true order of the system is six, and the system has three outputs, the number of observer Markov parameters that can be identified is two or greater, i.e., $p \geq 2$. For $p=2$, the identified observer Markov parameters are listed below.

$$
\begin{gathered}
\bar{Y}_{c}(1)=\left[\begin{array}{lllll}
0.2120 & 0.0014 & 1.8354 & 0.0326 & 0.0001 \\
0.0014 & 0.2139 & 0.0329 & 1.8254 & 0.0337 \\
0.0000 & 0.0007 & 0.0001 & 0.0164 & 1.9312
\end{array}\right] \\
\bar{Y}_{c}(2)=\left[\begin{array}{rrrrr}
-0.2120 & -0.0014 & -0.8969 & -0.0020 & 0.0000 \\
-0.0014 & -0.2139 & -0.0021 & -0.8874 & -0.0029 \\
0.0000 & -0.0007 & 0.0000 & -0.0011 & -0.9774
\end{array}\right]
\end{gathered}
$$


The two identified observer Markov parameters completely describe the closed-loop system, from which any number of closed-loop system Markov parameters can be computed. The closed-loop Markov parameters are the closed-loop system pulse response samples. From the computed closed-loop system Markov parameters and the controller gain, the open-loop system Markov parameters can be recovered. The open-loop system Markov parameters are the open-loop system pulse response samples. The calculation is illustrated here for the first few Markov parameters.

First, the closed-loop Markov parameters $Y_{c}(1), Y_{c}(2), Y_{c}(3), \ldots$ are computed using Eq. (21)

$$
\begin{gathered}
Y_{c}(1)=\bar{Y}_{c}^{(1)}(1)=\left[\begin{array}{ll}
0.2120 & 0.0014 \\
0.0014 & 0.2139 \\
0.0000 & 0.0007
\end{array}\right] \\
Y_{c}(2)=\bar{Y}_{c}^{(1)}(2)+\bar{Y}_{c}^{(2)}(1) Y_{c}(1)=\left[\begin{array}{ll}
0.1771 & 0.0081 \\
0.0081 & 0.1776 \\
0.0000 & 0.0042
\end{array}\right] \\
Y_{c}(3)=\bar{Y}_{c}^{(2)}(1) Y_{c}(2)+\bar{Y}_{c}^{(2)}(2) Y_{c}(1)=\left[\begin{array}{ll}
0.1353 & 0.0190 \\
0.0190 & 0.1330 \\
0.0002 & 0.0100
\end{array}\right], \ldots
\end{gathered}
$$

where $\bar{Y}_{c}^{(1)}(1), \bar{Y}_{c}^{(2)}(1)$ and $\bar{Y}_{c}^{(1)}(2), \bar{Y}_{c}^{(2)}(2)$ are the $3 \times 2$ and $3 \times 3$ partitions of $\bar{Y}_{c}(1)$ and $\bar{Y}_{c}(2)$, respectively. Beginning with $Y_{c}(3)$, the extra closed-loop Markov parameters are computed by setting $\bar{Y}_{c}(i)=0, i=3,4, \ldots$. Next, the open-loop system Markov parameters $Y(1), Y(2), Y(3)$, ... can be computed using Eq. (28).

$$
\begin{gathered}
Y(1)=Y_{c}(1)=\left[\begin{array}{ll}
0.2120 & 0.0014 \\
0.0014 & 0.2139 \\
0.0000 & 0.0007
\end{array}\right] \\
Y(2)=Y_{c}(2)-Y_{c}(1) F Y(1)=\left[\begin{array}{ll}
0.1940 & 0.0084 \\
0.0084 & 0.1995 \\
0.0000 & 0.0043
\end{array}\right]
\end{gathered}
$$




$$
Y(3)=Y_{c}(3)-Y_{c}(1) F Y(2)-Y_{c}(2) F Y(1)=\left[\begin{array}{ll}
0.1648 & 0.0208 \\
0.0208 & 0.1733 \\
0.0002 & 0.0106
\end{array}\right], \ldots
$$

In the same fashion, all desired closed-loop and open-loop system Markov parameters can be computed from the two identified observer Markov parameters. The obtained results can be verified using the open-loop model given in Egs. (110) and the controller gain given in Eq. (112). This example illustrates the case where the number of observer Markov parameters to be identified is chosen to be $2, p=2$, which is the minimum number for this case. The same result is obtained if more observer Markov parameters are identified. The same computation procedure still applies with the exception that the observer Markov parameters are now set to zero at a late time step. For example, say for $p=3$, the observer Markov parameters $\bar{Y}_{c}(i)$ are set to zero for $i=4,5, \ldots$

Shown in Fig. 4 is the closed-loop pulse response function of the second-output first-input pair obtained from the first 200 closed-loop Markov parameters computed from the two identified observer Markov parameters. Results for the other input-output pairs are similar although they are not shown here. Shown in Fig. 5 is the result obtained from the first 200 open-loop Markov parameters that are computed from the 200 closed-loop Markov parameters and the controller gain.

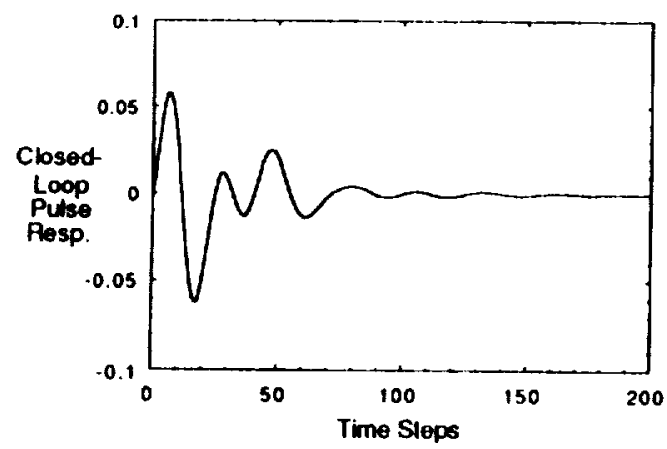

Fig. 4: Identified closed-loop pulse resp. (Closed-loop Markov parameters)

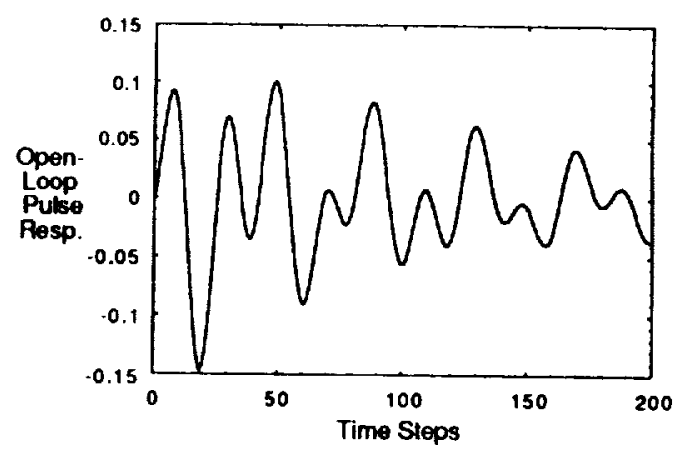

Fig. 5: Identified open-loop pulse resp. (Open-loop Markov parameters)

From the identified open-loop Markov parameters, a state space realization for the system can be found via realization theory. 
Example 2: To illustrate the closed-loop identification problem with the direct transmission term, consider the same system in Example 1, with the exception that the direct transmission term is now non-zero.

$$
D=\left[\begin{array}{rr}
0.1165 & 0.0352 \\
0.0627 & -0.0697 \\
0.0075 & 0.1696
\end{array}\right]
$$

The closed-loop system is excited by the perturbing the output signal the same as before. Also, let $p=2$, the identified observer Markov parameters are listed below.

$$
\begin{gathered}
\bar{Y}_{c}(0)=\left[\begin{array}{rr}
0.1165 & 0.0352 \\
0.0627 & -0.0697 \\
0.0075 & 0.1696
\end{array}\right] \quad \bar{Y}_{c}(1)=\left[\begin{array}{rrrrr}
-0.0132 & -0.0636 & 1.9199 & 0.0651 & 0.0001 \\
-0.1239 & 0.3416 & 0.0799 & 1.7616 & 0.0337 \\
-0.0156 & -0.0156 & 0.0059 & 0.1796 & 1.9312
\end{array}\right] \\
\bar{Y}_{c}(2)=\left[\begin{array}{ccccc}
-0.0981 & 0.0327 & -0.9396 & -0.0191 & -0.0000 \\
0.0613 & -0.2826 & -0.0256 & -0.8531 & -0.0029 \\
0.0074 & 0.1650 & -0.0028 & -0.0840 & -0.9774
\end{array}\right]
\end{gathered}
$$

The direct transmission term $D$ is simply $\bar{Y}_{c}(0)$. All open-loop system Markov parameters can be computed from $\bar{Y}_{c}(1), \bar{Y}_{c}(2)$, and the controller gain $F$ using Eqs. (52) and (54). With the direct transmission term, however, the computation is slightly more complicated. For purpose of illustration, the computation of the Markov parameters $C B, C A B$, and $C A^{2} B$ is shown below.

First, the closed-loop Markov parameters $Y_{c}(1), Y_{c}(2), Y_{c}(3), \ldots$ are computed using Eq. (52)

$$
\begin{gathered}
Y_{c}(1)=\bar{Y}_{c}^{(1)}(1)+\bar{Y}_{c}^{(2)}(1)(I-D F)^{-1} Y_{c}(0)=\left[\begin{array}{rr}
0.2032 & -0.0013 \\
-0.0053 & 0.2217 \\
-0.0000 & 0.0007
\end{array}\right] \\
Y_{c}(2)=\bar{Y}_{c}^{(1)}(2)+\bar{Y}_{c}^{(2)}(1)(I-D F)^{-1} Y_{c}(1)+\bar{Y}_{c}^{(2)}(2)(I-D F)^{-1} Y_{c}(0)=\left[\begin{array}{cc}
0.1702 & 0.0064 \\
0.0028 & 0.1821 \\
-0.0001 & 0.0043
\end{array}\right]
\end{gathered}
$$




$$
Y_{c}(3)=\bar{Y}_{c}^{(2)}(1)(I-D F)^{-1} Y_{c}(2)+\bar{Y}_{c}^{(2)}(2)(I-D F)^{-1} Y_{c}(1)=\left[\begin{array}{cc}
0.1302 & 0.0184 \\
0.0150 & 0.1362 \\
-0.0001 & 0.0104
\end{array}\right], \ldots
$$

where $\bar{Y}_{c}^{(1)}(1), \bar{Y}_{c}^{(2)}(1)$ and $\bar{Y}_{c}^{(1)}(2), \bar{Y}_{c}^{(2)}(2)$ are the $3 \times 2$ and $3 \times 3$ partitions of $\bar{Y}_{c}(1)$ and $\bar{Y}_{c}(2)$, respectively, and $\bar{Y}_{c}(i)=0, i=3,4, \ldots$ Second, the open-loop system Markov parameters are computed from the closed-loop Markov parameters above using Eq. (54). One thus obtains

$$
\begin{gathered}
Y(1)=Y_{c}(1)(I-F D)=\left[\begin{array}{ll}
0.2120 & 0.0014 \\
0.0014 & 0.2139 \\
0.0000 & 0.0007
\end{array}\right] \\
Y(2)=Y_{c}(2)(I-F D)-Y_{c}(1) F Y(1)=\left[\begin{array}{ll}
0.1940 & 0.0084 \\
0.0084 & 0.1995 \\
0.0000 & 0.0043
\end{array}\right] \\
Y(3)=Y_{c}(3)(I-F D)-Y_{c}(1) F Y(2)-Y_{c}(2) F Y(1)=\left[\begin{array}{ll}
0.1648 & 0.0208 \\
0.0208 & 0.1733 \\
0.0002 & 0.0106
\end{array}\right], \ldots
\end{gathered}
$$

The above identified open-loop Markov parameters can be easily verified by direct substitution.

Example 3: This example illustrates the case of closed-loop identification with an existing closed-loop dynamic controller. Consider the case where the 6-th order system in Example 2 is controlled by a 4-th order dynamic controller. The controller matrices are given below.

$$
\begin{aligned}
& P=\left[\begin{array}{cccc}
0.3392 & 0.0054 & 0.0000 & 0.0742 \\
0.0054 & 0.3391 & 0.0054 & 0.0005 \\
0.0000 & 0.0027 & 0.3419 & 0.0000 \\
-0.0986 & 0.0488 & 0.0003 & 0.3310
\end{array}\right], \quad Q=\left[\begin{array}{ccc}
0.0654 & -0.0071 & -0.0024 \\
-0.0335 & -0.0708 & -0.0419 \\
0.0303 & -0.0667 & -0.0402 \\
-0.0189 & 0.0517 & 0.0452
\end{array}\right] \\
& R=\left[\begin{array}{llll}
-0.0004 & 0.0031 & 0.0032 & 0.0030 \\
0.0040 & 0.0060 & -0.0106 & 0.0089
\end{array}\right], \quad S=\left[\begin{array}{ccc}
0.0191 & -0.0072 & 0.0083 \\
-0.0624 & -0.0085 & -0.0134
\end{array}\right]
\end{aligned}
$$

The closed-loop system is excited by a random perturbation. The time histories of the excitation signal $v_{1}(i), v_{2}(i)$, the system input $u(i)$, the system output $y(i)$, and the controller state $s(i)$, each 
of 100 data samples, are recorded for system identification purposes. Since the dimension of the augmented state is $n+n_{s}=6+4=10$, and the dimension of the augmented output is $q+n_{s}=3+4=7$, the minimum number of observer Markov parameters that are to be identified is two in the identification algorithm, $p=2$. For $p=2$, the identified observer Markov parameter matrix is of dimensions $7 \times 32$. From this matrix, any number of closed-loop Markov parameters for the augmented system can be computed. For illustration, the first few Markov parameters are computed below using Eq. (89).

$$
\begin{gathered}
Y_{a c}(0)=\bar{Y}_{a c}(0)=D_{a}=\left[\begin{array}{ccccccc}
0.1165 & 0.0352 & 0 & 0 & 0 & 0 \\
0.0627 & -0.0697 & 0 & 0 & 0 & 0 \\
0.0075 & 0.1696 & 0 & 0 & 0 & 0 \\
0 & 0 & 0 & 0 & 0 & 0 \\
0 & 0 & 0 & 0 & 0 & 0 \\
0 & 0 & 0 & 0 & 0 & 0 \\
0 & 0 & 0 & 0 & 0 & 0
\end{array}\right] \\
Y_{a c}(1)=\bar{Y}_{a c}^{(1)}(1)+\bar{Y}_{a c}^{(2)}(1)\left(I-D_{a} F_{a}\right)^{-1} Y_{a c}(0)=\left[\begin{array}{ccccccc}
0.2124 & 0.0019 & 0 & 0 & 0 & 0 \\
-0.0003 & 0.2131 & 0 & 0 & 0 & 0 \\
-0.0000 & 0.0007 & 0 & 0 & 0 & 0 \\
0.0072 & 0.0024 & 1 & 0 & 0 & 0 \\
-0.0087 & -0.0034 & 0 & 1 & 0 & 0 \\
-0.0009 & -0.0011 & 0 & 0 & 1 & 0 \\
0.0014 & 0.0034 & 0 & 0 & 0 & 1
\end{array}\right]
\end{gathered}
$$

Using the fact that $\bar{Y}_{\alpha c}(i) \equiv 0, i=3,4, \ldots$, the remaining closed-loop Markov parameters of the augmented system can be computed as follows.

$$
\begin{aligned}
Y_{\alpha c}(2) & =\bar{Y}_{a c}^{(1)}(2)+\bar{Y}_{a c}^{(2)}(1)\left(I-D_{a} F_{a}\right)^{-1} Y_{a c}(1)+\bar{Y}_{a c}^{(2)}(2)\left(I-D_{a} F_{a}\right)^{-1} Y_{a c}(0) \\
& =\left[\begin{array}{cccccc}
0.1951 & 0.0085 & -0.0001 & 0.0007 & 0.0006 & 0.0006 \\
0.0040 & 0.1984 & 0.0009 & 0.0013 & -0.0023 & 0.0019 \\
0.0000 & 0.0042 & 0.0000 & 0.0000 & 0.0000 & 0.0000 \\
0.0164 & -0.0004 & 0.3392 & 0.0054 & 0.0000 & 0.0742 \\
-0.0100 & -0.0163 & 0.0054 & 0.3391 & 0.0054 & 0.0004 \\
0.0061 & -0.0146 & 0.0000 & 0.0027 & 0.3419 & 0.0000 \\
-0.0047 & 0.0117 & -0.0986 & 0.0488 & 0.0003 & 0.3311
\end{array}\right]
\end{aligned}
$$




$$
\begin{aligned}
Y_{\alpha c}(3) & =\bar{Y}_{a c}^{(2)}(1)\left(I-D_{a} F_{a}\right)^{-1} Y_{c}(2)+\bar{Y}_{a c}^{(2)}(2)\left(I-D_{a} F_{a}\right)^{-1} Y_{a c}(1) \\
& =\left[\begin{array}{cccccc}
0.1663 & 0.0205 & -0.0001 & 0.0009 & 0.0008 & 0.0009 \\
0.0142 & 0.1718 & 0.0009 & 0.0017 & -0.0028 & 0.0025 \\
0.0001 & 0.0105 & 0.0000 & 0.0000 & 0.0000 & 0.0000 \\
0.0179 & -0.0002 & 0.1078 & 0.0073 & 0.0001 & 0.0498 \\
-0.0101 & -0.0201 & 0.0036 & 0.1149 & 0.0038 & 0.0005 \\
0.0077 & -0.0182 & 0.0000 & 0.0018 & 0.1171 & -0.0001 \\
-0.0072 & 0.0134 & -0.0658 & 0.0322 & 0.0003 & 0.1024
\end{array}\right], \ldots
\end{aligned}
$$

Using Eq. (90), the Markov parameters $Y_{a}(i)$ are then computed.

$$
\begin{aligned}
& Y_{a}(1)=Y_{a c}(1)\left(I-F_{a} D_{a}\right)=\left[\begin{array}{cccccc}
0.2120 & 0.0014 & 0 & 0 & 0 & 0 \\
0.0014 & 0.2139 & 0 & 0 & 0 & 0 \\
0.00 \times 0) & 0.0007 & 0 & 0 & 0 & 0 \\
0 & 0 & 1 & 0 & 0 & 0 \\
0 & 0 & 0 & 1 & 0 & 0 \\
0 & 0 & 0 & 0 & 1 & 0 \\
0 & 0 & 0 & 0 & 0 & 1
\end{array}\right] \\
& Y_{a}(2)=Y_{a c}(2)\left(I-F_{a} D_{a}\right)-Y_{a c}(1) F_{a} Y_{a}(1)=\left[\begin{array}{cccccc}
0.1940 & 0.0084 & 0 & 0 & 0 & 0 \\
0.0084 & 0.1995 & 0 & 0 & 0 & 0 \\
0.0000 & 0.0043 & 0 & 0 & 0 & 0 \\
0 & 0 & 0 & 0 & 0 & 0 \\
0 & 0 & 0 & 0 & 0 & 0 \\
0 & 0 & 0 & 0 & 0 & 0 \\
0 & 0 & 0 & 0 & 0 & 0
\end{array}\right] \\
& Y_{a}(3)=Y_{\alpha c}(3)\left(I-F_{a} D_{a}\right)-Y_{a c}(1) F_{a} Y_{a}(2)-Y_{a c}(2) F_{a} Y_{a}(1)=\left[\begin{array}{cccccc}
0.1648 & 0.0208 & 0 & 0 & 0 & 0 \\
0.0208 & 0.1733 & 0 & 0 & 0 & 0 \\
0.0002 & 0.0106 & 0 & 0 & 0 & 0 \\
0 & 0 & 0 & 0 & 0 & 0 \\
0 & 0 & 0 & 0 & 0 & 0 \\
0 & 0 & 0 & 0 & 0 & 0 \\
0 & 0 & 0 & 0 & 0 & 0
\end{array}\right] \cdots(137)
\end{aligned}
$$


Again, it can be verified that the upper left partitions of $\bar{Y}_{a c}(0), Y_{a}(1), Y_{a}(2), Y_{a}(3), \ldots$ are the open-loop system Markov parameters $D, C B, C A B, C A^{2} B, \ldots$ respectively.

\section{Concluding Remarks}

This paper develops a procedure for identification of open-loop systems operating in the closedloop. First, the basic problem of identifying the open-loop system with an existing linear output feedback controller is formulated. The approach used here is to inject additive excitation during closed-loop operation, and closed-loop data is used for identification. The procedure consists of two basic steps. First, through an associated observer, the closed-loop system Markov parameters are identified. Second, the open-loop system is recovered from the identified closed-loop parameters. Relations between the identified observer Markov parameters, the closed-loop system Markov parameters, and the open-loop system Markov parameters are established. The basic formulation is extended to the case of closed-loop identification of a system with the direct transmission term. This case is important since accelerometers are often used as measurement devices on large flexible structures. The considerably more complicated case of closed-loop identification of a system with an existing dynamic feedback controller is also treated in this paper. The developed solution requires perturbation of both the input signal and the controller state for identification. Under this condition, it is shown that the identification problem with an existing dynamic controller can be formulated to mimic the problem of identification with an linear output feedback controller of an augmented dynamic system, which has a simple closed-form solution. For the case of two cascading linear systems, it is sometimes possible to recover the Markov parameters of one system when the Markov parameters of the other system and those of the combined system are known. Numerical examples are provided to illustrate the identification procedure for each of the respective closed-loop cases.

\section{References}

1 Phan, M., Juang, J.-N., and Longman, R.W., "On Markov Parameters in System Identification, " NASA Technical Memorandum, TM 104156, October 1991. 
2 Chen, C.-W., Huang, J.-K., Phan, M. and Juang, J.-N., "Integrated System Identification and Modal State Estimation for Control of Large Flexible Space Structures," Journal of Guidance, Control, and Dynamics, Vol. 15, No. 1, pp. 88-95, Jan.-Feb. 1992.

3 Phan, M., Juang, J.-N., and Longman, R.W., "Identification of Linear Multivariable Systems by Identification of Observers with Assigned Real Eigenvalues," The Journal of the Astronautical Sciences, Vol. 40, No. 2, April-June 1992 (to appear).

4 Juang, J.-N., Phan, M., Horta, L.G., and Longman, R.W., "Identification of Observer/Kalman Filter Markov Parameters: Theory and Experiment," Proceedings of the AIAA Guidance, Navigation, and Control Conference, New Orleans, Louisiana, August 1991.

5 Phan, M., Horta, L.G., Juang, J.-N., and Longman, R.W., "Linear System Identification Via An Asymptotically Stable Observer " Proceedings of the AIAA Guidance, Navigation, and Control Conference, New Orleans, Louisiana, August 1991. Also, to appear in the Journal of Optimization Theory and Applications.

6 Horta, L.G., Phan, M., Juang, J.-N., Longman, R.W., and J.L. Sulla, "Frequency Weighted System Identification and Linear Quadratic Controller Design," Proceedings of the AIAA Guidance, Navigation, and Control Conference, New Orleans, Louisiana, August 1991.

7 Phan, M., Horta, L.G., Juang, J.-N., and Longman, R.W., "Improvement of Observer/Kalman Filter Identification by Residual Whitening," submitted for possible presentation at the AIAA Guidance, Navigation, and Control Conference, Hilton Head, South Carolina, August 1992.

8 Phan, M., Horta, L.G., Juang, J.-N., and Longman, R.W., "Identification of Linear Systems Via An Asymptotically Stable Observer " NASA Technical Paper 3164, 1992.

9 Kabamba, P.T. and Longman, R.W., "An Integrated Approach to Optimal Reduced Order Control Theory," Proceedings of the 1988 VPI\&SUIAIAA Symposium on Dynamics and Control of Large Structures, May 1988.

10 Juang, J.-N. and Pappa, R.S., "An Eigensystem Realization Algorithm for Modal Parameter Identification and Model Reduction," Journal of Guidance, Control, and Dynamics, Vol. 8, September-October 1985.

11 Longman, R.W. and Juang, J.-N., "A Variance Based Confidence Criterion for ERA Identified Modal Parameters," Proceedings of the AASIAIAA Astrodynamics Specialist Conference, Kalispell, Montana, August 1987.

12 Juang, J.-N. and Phan, M., "Identification of System, Observer, Controller from ClosedLoop Experimental Data," submitted for possible presentation at the AIAA Guidance, Navigation, and Control Conference, Hilton Head, South Carolina, August 1992. 


\section{REPORT DOCUMENTATION PAGE}

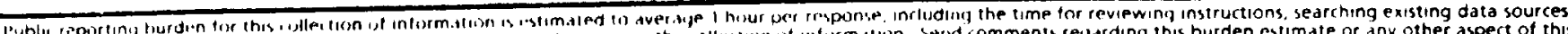
Publi reporfinif

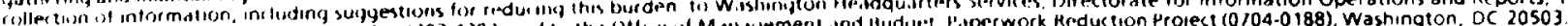

\begin{tabular}{ll|l}
\hline 1. AGENCY USE ONLY (Leave blank) & 2. REPORT DATE & 3. REPORT TYPE AND DATES COVERED
\end{tabular}

\begin{tabular}{l|l|l} 
1. AGENCY USE ONLY (Leave blank) & $\begin{array}{l}\text { 2. REPORr DAYE } \\
\text { April } 1992\end{array}$ & Technical Memorandum
\end{tabular}

\section{TITLE AND SUBTITLE}

System Identification from Closed-Loop Data with Known output Feedback Dynamics

\section{FUNDING NUMBERS}

WU 590-14-21-01

\section{AUTKOR(S)}

Minh Phan, Jer-Nan Juang, lucas G. Horta, and Richard W. longman

\section{PERFORMING ORGANIZATION NAME(S) AND ADDRESS(ES)}

NASA langley Research Center

Hampton, VA 23665-5225

\section{SPONSORING/MONITORING AGENCY NAME(S) AND ADDRESS(ES)}

National Aeronatics and Space Administration

Washington, DC 20546-0001

8. PERFORMING ORGANIZATION REPORT NUMBER

\section{SUPPLEMENTARY NOTES}

Phan: lockheed Englneering \& Sciences Co., Hampton, VA; Juang: Langley Research center, Hampton, VA; Horta: Langley Research Center, Hampton, VA; Longman: Columbia University, New York, NY

12a. DISTRIBUTION / AVAILABILITY STATEMENT

12b. DISTRIBUTION CODE

Unclassified--Unlimited

Subject Category 39

\section{ABSTRACT (Maximum 200 words)}

This paper presents a procedure to identify the open-loop system when it is operating under closed-loop conditions. First, closed-loop excitation data are used to compute the system open-loop and closed-loop Markov parameters. The Markov parameters, which are the pulse response simples, are then used to compute a state space representation of the open-loop system. 'lwo closed-loop conligurations are considered in this paper: The closed-loop system can either have a linear output feedback controller or a dynamic output feedback controller. Numerical examples are provided to illustrate the proposed closed-loop identification method.

\section{SUBJECT TERMS}

Closed-loop ldentification, linear Output liecdback, Dynamic output Feedback

17. SECURITY CLASSIFICATION
OF REPORT
Unc lassif ied

Unclassified

18. SECURITY CLASSIFICATION
OF THIS PAGE
Unc lassif ied

19. SECURITY CLASSIFICATION OF ABSTRACT
15. NUMBER OF PAGES 33

16. PRICE CODE $\mathrm{AO} 3$

20. LIMITATION OF ABSTRACT 

